\title{
Fluctuating asymmetry does not increase with moderate inbreeding in Drosophila melanogaster
}

\author{
KEVIN FOWLER* \& MICHAEL C. WHITLOCK \\ Institute of Cell, Animal, and Population Biologv, University of Edinburgh, West Mains Road, Edinburgh EH9 3JT, U.K.
}

\begin{abstract}
Fluctuating asymmetry, the unsigned difference between character values on the left and right sides of an individual, is often thought to be highly correlagted with the heterozygosity of individuals or populations. A large sample of Drosophila melanogaster individuals with an inbreeding coefficient of $F=0.25$ was derived from a laboratory population and compared to a sample of outbred individuals for the fluctuating asymmetry of sternopleural bristle number. Inbred flies were not more asymmetric than outbred flies. There was -no evidence for heritability of fluctuating asymmetry, as measured by variance among full-sib lines. Fluctuating asymmetry may not be a reliable measure of the degree of inbreeding at the relatively low levels found in most animal populations and should be used with caution in the management of endangered species.
\end{abstract}

Keywords: Drosophila melanogaster, fluctuating asymmetry, inbreeding, sternopleural bristles.

\section{Introduction}

When fitness depends upon the phenotypic values of particular characters, organisms which can produce those phenotypes reliably should be more fit than those which are less buffered against the effects of environmental disturbances during development. This developmental stability has often been thought to be associated with heterosis and to be one cause of inbreeding depression (Lerner, 1954; Waddington, 1960, 1966). As such, the instability of development under environmental noise has been thought to be an indicator of the amount of homozygosity in a population (Wayne et al., 1986; Modi et al., 1987; Quattro \& Vrijenhoek, 1989; Leary \& Allendorf, 1989; Parsons, 1990, 1992).

One measure of developmental stability that has been used frequently is fluctuating asymmetry (FA), the nondirectional deviation from bilateral symmetry (Van Valen, 1962; Palmer \& Strobeck, 1986). FA is a useful measure of developmental stability, because generally there is little or no genetic variance for unsigned differences between the sides. Therefore, any deviations from one side to another must be due to different responses to environmental noise (Palmer \& Strobeck, 1986). Furthermore, we know that the optimal pheno-

${ }^{*}$ Correspondence: Department of Genetics and Biometry, University College London, 4 Stephenson Way, London NW1 2HE, U.K. type for many characters is perfect symmetry; that is, the most fit individuals have the smallest FA values. We do not know, however, the strength of selection against such deviations. FA is therefore one of the best possible measures of developmental stability and canalization.

In view of the notion that heterozygosity has been thought to promote developmental stability (Lerner, 1954; Soulé, 1979), many studies have looked for a positive correlation between FA and homozygosity, either of individuals or of populations. In several early studies, severely inbred lines were compared to the $F_{1}$ cross between such lines (Robertson \& Reeve, 1952; Mather, 1953; Reeve, 1960; Beardmore, 1960). In general, the $F_{1}$ individuals had lower asymmetry values than the inbred lines from which they were derived.

More recently, with the advent of electrophoretic analyses, the isozyme heterozygosity of individuals and populations has been examined in relation to FA in a wide variety of species: bivalves (Kat, 1982); mice (Leamy, 1984); Drosophila melanogaster (Houle, 1989); salmonid fishes (Leary et al., 1983, 1984, 1985); cheetah (Kieser \& Groeneveld, 1991); poeciliid fishes (Vrijenhoek \& Lerman, 1982; Quattro \& Vrijenhoek, 1989); forked fungus beetles (Whitlock, 1993); and side-blotched lizards (Soulé, 1979), for example. Several cases are reviewed in Palmer \& Strobeck (1986). The pattern of results is variable. In most of these cases there were significant correlations 
of FA and heterozygosity; but in four cases (Houle, 1989; Leary et al., 1984; Kieser \& Groeneveld, 1991; Whitlock, 1993), no significant relationship was found.

However, there is a problem that is common to many analyses of enzyme-associated heterozygosity. Predictions of total heterozygosity based on measures at a handful of loci will be unreliable for species which are typically outbred (Chakraborty, 1987). Such measures do have more predictive power if there is variance in the degree of inbreeding within a sample.

There is a third method of comparing the degree of developmental stability among individuals of different degrees of homozygosity. Experimental manipulation of the mating structure of populations allows the direct comparison of the FA values of different groups inbred to varying degrees. This method avoids the problems of estimating homozygosity inherent in the isozyme analysis, yet allows us to examine the effects of homozygosity at more natural levels. There has been a single study of this type which concluded that there was no significant correlation between the degree of inbreeding and FA (Clark et al., 1992). In this study, we compare the level of FA in an outbred line of Drosophila melanogaster with that in a group of 30 lines inbred to a relative inbreeding coefficient of $F=0.25$.

\section{Materials and methods}

\section{Stocks}

We used a laboratory stock of $D$. melanogaster collected in 1970 in Dahomey (now Benin) and maintained since then at a large population size in population cage culture. This stock shows considerable phenotypic and genetic variation for a wide range of characters, including sternopleural bristle numbęr (Wilkinson et al., 1990). All flies were kept at $25 \pm 1{ }^{\circ} \mathrm{C}$ with a fixed illumination cycle of $12 \mathrm{~h}$ light followed by $12 \mathrm{~h}$ dark. All handling was performed at room temperature using carbon dioxide anaesthesia.

We obtained a large sample of eggs from the Dahomey stock in four unyeasted culture bottles placed in the cage for $6 \mathrm{~h}$. The progeny from each bottle were collected as virgins and a random sample of 100 males and 100 females were mated in pairs. The $F_{1}$ progeny of these pair-matings (families) were used to set up two types of line; an 'outbred' and an 'inbred' line. The outbred stock was obtained by mixing together groups of five males and five females from each family to produce a total sample of 1008 flies. Random groups of 18 males and 18 females from this sample were allocated to each of 28 culture bottles. Thirty inbred lines were produced by choosing 30 families at random and allocating 18 males and 18 females from each family to each of four culture bottles. The estimated inbreeding coefficient of the outbred line relative to the cage populations was therefore approximately $F=0.0005$, while the inbreeding coefficient of each of the inbred lines was approximately $F=0.25$. These inbred and outbred lines were maintained with random mixing among bottles of each line in the $F_{2}$ generation, before measurements were taken on the $\mathrm{F}_{3}$ generation.

\section{Measurements}

Flies to be measured were raised at constant density, with a very small variance in age. $F_{2}$ flies were allowed to lay eggs for a $2 \mathrm{~h}$ period on grape juice medium (Fowler \& Partridge, 1986). After 25 h, 50 first instar larvae were transferred on paint brushes from this medium to each of four standard food vials for each inbred line and to 26 vials for the outbred line. After emergence of all flies, the progeny from each vial were placed in small vials and frozen at $-70^{\circ} \mathrm{C}$. Subsequently, sternopleural bristles were counted on each side of the fly for 352 flies of each sex for the control lines, and between 32 and 94 flies from each sex in each inbred line. In total, 1675 females and 1683 males were assayed for the inbred lines.

\section{Analysis}

Fluctuating asymmetry was calculated for sternopleural bristle counts for each group as

$\mathrm{FA}=\operatorname{var}\left[\frac{R-L}{(L+R) / 2}\right]$,

as recommended by Palmer \& Strobeck (1986) as the most efficient test $(R$ and $L$ indicate the values on the right and left sides of the individuals, respectively). This measure of FA is the most powerful measure for detecting difference between groups when the magnitude of FA scales with the character mean (Palmer \& Strobeck, 1986).

The FA value was calculated by sex for all inbreds and all outbreds, as well as for each inbred line individually. The FA values for each sex for inbreds and outbreds were compared by a one-tailed $F$-test. Variance among inbred lines for FA values was tested by Bartlett's test for the homogeneity of variances (Sokal \& Rohlf, 1981).

\section{Results}

Among the controls, the mean numbers of sternopleural bristles were 20.6 in males and 21.5 in females and the variances were 7.8 and 6.8 , respectively. The means in the inbred lines were 20.5 and 21.6 which are 
not statistically distinguishable from the control means. The average variance within inbred lines for sternopleural bristle number was not statistically different from the additive, neutral expectation of $\left(1-h^{2} F\right)$ times the variance in the outbred population, where $h^{2}$ is the heritability of the trait $(0.80)$. The average variance within inbred lines was 6.0 and 6.1 (Whitlock \& Fowler, submitted).

The FA values for males, females and both sexes combined for outbred and inbred lines are given in Table 1. As can be seen, the values of FA are extremely close for both inbred and outbred flies. The FA for inbred males is even slightly smaller than that for outbred males. The very high $P$ values of the $F$-tests emphasize the lack of differences between inbreeding levels, even with the relatively large data sets available here. Flies inbred to $F=0.25$ are not more asymmetric than outbred flies, when taken from the same genetic stock and measured under carefully controlled conditions.

There is no significant variance among full-sib lines in the amount of fluctuating asymmetry (Bartlett's test of homogeneity of variances; males: $\chi_{29}^{2}=32.7$, $P=0.29$; females: $\chi_{29}^{2}=31.2, P=0.36$ ). This indicates that there is little or no genetic variance or heritability for FA in either sex. There is considerable variance among lines in the mean number of bristles, for both males and females $(P<0.0001$, nested analysis of variance).

\section{Discussion}

The level of FA in a population has often been shown to be correlated with the amount of heterozygosity in that population. Here, we have used populations which have been inbred to a known degree, thereby eliminating the estimation error of heterozygosity, yet there is no significant relationship between level of inbreeding and the amount of fluctuating asymmetry in these populations. Clearly, even without the possible confusion over the error in measuring the amount of heterozygosity in a population attributable to isozyme analyses, there are characters in populations which do

Table 1 Values of fluctuating asymmetry for outbred and inbred Drosophila melanogaster

\begin{tabular}{llll}
\hline & Males & Females & $\begin{array}{c}\text { Sexes } \\
\text { combined }\end{array}$ \\
\hline Outbred & 0.0209 & 0.0194 & 0.0201 \\
Inbred & 0.0205 & 0.0206 & 0.0206 \\
$F$ & 0.982 & 1.06 & 1.02 \\
$P$ & 0.59 & 0.24 & 0.36 \\
\hline
\end{tabular}

not demonstrate the usual pattern. It is probably the case that other data showing negative results have gone unreported.

Previous studies have usually used lines which are extremely inbred and are crossed to produce an $\mathrm{F}_{1}$ 'outbred' line (Robertson \& Reeve, 1952; Mather, 1953; Reeve, 1960; Beardmore, 1960). These studies have often shown a significantly lower asymmetry in the outbred lines than in the inbred lines. However, in each case these results were based on only two inbred lines. Our results have the advantage of being based on 30 inbred lines. A further strength of our study is that it provides information about the effects of inbreeding at the less extreme level of $F=0.25$. Our results show that for sternopleural bristle number in $D$. melanogaster there is no relationship between FA and inbreeding at this intermediate level. This finding agrees with the results of Clark et al. (1992) for five wing morphology characters in Apis mellifera. They examined FA in relation to a range of known heterozygosities in this haplo-diploid species and found no association between these factors.

One reason for investigating this relationship at intermediate inbreeding levels is because of its importance in the context of conservation biology. FA has been suggested as a straightforward measure of the amount of genetic and environmental 'stress' affecting a particular species or population (Wayne et al., 1986; Modi et al., 1987; Quattro \& Vrijenhoek, 1989; Leary \& Allendorf, 1989; Parsons, 1992). Since species in need of management for conservation have inbreeding coefficients at intermediate values, we must determine whether the effects of inbreeding at these levels are reflected in differences in FA. In the case of this study, FA is clearly not increased by extra inbreeding. It may be possible to use fluctuating asymmetry as an indicator of the presence of stress in a population, but these results show that the absence of FA is no indication that a population has a high level of genetic heterozygosity (Whitlock, 1993).

There is also no evidence in this population for any genetic variance for the amount of asymmetry. Previous analyses in flies have shown that asymmetry can be increased by selection (Reeve, 1960; and see Palmer \& Strobeck, 1986), but the Dahomey sample demonstrates no significant variance among inbred lines for FA, despite a large heritability for the sternopleural bristle counts and other characters (results above and Wilkinson et al., 1990).

The use of FA as a measure of developmental stability has a long history. More precise evidence about the effects of intermediate levels of inbreeding and homozygosity on the amount of FA, provided by analysis of explicit breeding designs such as the one described here, give more information about the effects 
of homozygosity per se. Heterozygosity does not necessarily allow a more stable development.

\section{Acknowledgements}

We thank The Royal Society (K.F.) and SERC (M.C.W.) for financial support and an anonymous referee for useful comments on the manuscript.

\section{References}

BEARDMORE, J. A. 1960. Developmental stability in constant and fluctuating environments. Heredity, 14, 411-422.

CHAKRABORTY, R. 1987. Biochemical heterozygosity and phenotypic variability of polygenic traits. Heredity, $\mathbf{5 9}$, 19-28.

ClARK, G. M., OLDROYD, B. P. AND HUNT, P. 1992. The genetic basis of developmental stability in Apis mellifera: heterozygosity versus genic balance. Evolution, 46, 753-762.

FOWLER, K. AND PARTRIDGE, L. 1986. Variation in male fertility explains an apparent effect of genotypic diversity on success in larval competition in Drosophila melanogaster. Heredity, 57, 31-36.

HoULE, D. 1989. Allozyme-associated heterosis in Drosophila melanogaster. Genetics, 123, 789-801.

KAT, P. w. 1982. The relationship between heterozygosity for enzyme loci and developmental homeostasis in peripheral populations of aquatic bivalves (Unionidae). Am. Nat., 119, 824-832.

KIESER, J. A. AND GROENEVELD, H. T. 1991. Fluctuating odontometric asymmetry, morphological variability, and genetic monomorphism in the cheetah Acinonyx jubatus. Evolution, 45, 1175-1183.

LEAMY, L. 1984. Morphometric studies in inbred and hybrid house mice. V. Directional and fluctuating asymmetry. Am. Nat., 123, 579-593.

LEARY, R. F. AND ALLENDORF, F. w. 1989. Fluctuating asymmetry as an indication of stress: implications for conservation biology. Trends Ecol. Evol., 4, 214-217,

LEARY, R. F., ALLENDORF, F. W. AND KNUDSON, R. L. 1983. Developmental stability and enzyme heterozygosity in rainbow trout. Nature, 301, 71-72.

LEARY, R. F., ALLENDORF, F. W. AND KNUDSON, R. L. 1984. Superior developmental stability of heterozygotes of enzyme loci in salmonid fishes. Am. Nat., 124, 540-551.
LEARY, R. F., ALLENDORF, F. W. AND KNUDSON, R. L. 1985. Inheritance of meristic variation and the evolution of developmental stability in rainbow trout. Evolution, 39, 308-314.

LerNer, I. M. 1954. Genetic Homeostasis. Oliver and Boyd, Edinburgh.

MATHER, K. 1953. Genetical control of stability in development. Heredity, 7, 297-336.

MODI, W. S., WAYNE, R. K. AND O'BRIEN, S. J. 1987. Analysis of fluctuating asymmetry in cheetahs. Evolution, 41, 227-228.

PALMER, A. R. AND STROBECK, C. 1986. Fluctuating asymmetry: measurement, analysis, patterns. Ann. Rev. Ecol. Syst., 17, 391-421.

PARSONS, P. A. 1990. Fluctuating asymmetry and stress intensity. Trends Ecol. Evol., 5, 97-98.

PARSONS, P. A. 1992. Fluctuating assymetry: a biological monitor of environmental and genetic stress. Heredity, 68, 361-364.

REEVE, E. C. R. 1960. Some genetic tests on asymmetry of sternopleural chaeta number in Drosophila. Genet. Res., 1, 151-172.

ROBERTSON, F. W. AND REEVE, E. C. R. 1952. Heterozygosity, environmental variation and heterosis. Nature, 170, 286.

QUATTRO, J. M. AND VRIJENHOEK, R. C. 1989. Fitness differences among remnant populations of the endangered Sonoran topminnow. Science, 245, 976-978.

SOKAL, R. R. AND ROHLF, F. J. 1981. Biometry, 2nd edn. Freeman, San Francisco.

SOULE, M. E. 1979. Heterozygosity and developmental stability: another look. Evolution, 33, 396-401.

VAN VALEN, L. 1962. A study of fluctuating asymmetry. Evolution, 16, 125-142.

VRIJENHOEK, R. C. AND LERMAN, S. 1982. Heterozygosity and developmental stability under sexual and asexual breeding systems. Evolution, 36, 768-776.

WADDINGTON, C. H. 1960. Experiments in canalizing selection. Genet. Res., 1, 140-150.

Waddington, C. H. 1966. Principles of Development and Differentiation. Macmillan, New York.

WAYNE, R. K., MODI, W. S. AND O'BRIEN, S. J. 1986. Morphological variability and asymmetry in the cheetah Acinonyx jubatus. Evolution, 40, 78-85.

WHITLOCK, M. C. 1993. Lack of correlation between heterozygosity and fitness in forked fungus beetles. Heredity, 70, 574-581.

WHITLOCK, M. C. AND FOWLER, $\mathrm{K}$. The distribution among populations in phenotypic variance with inbreeding. Evolution (submitted).

WILKINSON, G. S., FOWLER, K. AND PARTRIDGE, L. 1990. Resistance of genetic correlation structure to directional selection in Drosophila melanogaster. Evolution, 44, 1990-2003. 